\title{
BINOCULAR BRIGHTNESS AVERAGING AND CONTOUR INFORMATION
}

\author{
BY W. J. M. LEVELT \\ Institute for Perception RVO-TNO, Soesterberg, The Netherlands
}

\begin{abstract}
Binocular brightness averaging has been investigated under two conditions: with identical contour information in both eyes and with different contour information.

Equibrightness curves are presented for the simple case, in which right and loft test fields are identical in pattern but different in luminance. These curves are for the most part linear; i.e. if the weighted sum of left and right luminance is constant, the same binoeular brightness impression is produced. The sum of the weighting co-efficients is unity (law of complementary shares). In the absence of eye dominance, the weights are equal; otherwise a correction for eye dominence must be made.

If monocular contour information is present in one test field, brightnoss averaging remainx linear, but the weight for that eye inereases at the cost of the weight for the other eye. In a region close to a monoeular contour (within $1^{\circ}$ of visual anglo), the weight approaches unity, so that binocular brightness in this region is dependent upon the luminance in one eye only.

A suggested explanation of Fechner's paradox is given, and the implications of the approach for the mechanism of binocular rivalry are considered.
\end{abstract}

The present paper describes a number of experiments on binocular brightness averaging, and on the influence of contour information in one eye upon binocular brightness interaction.

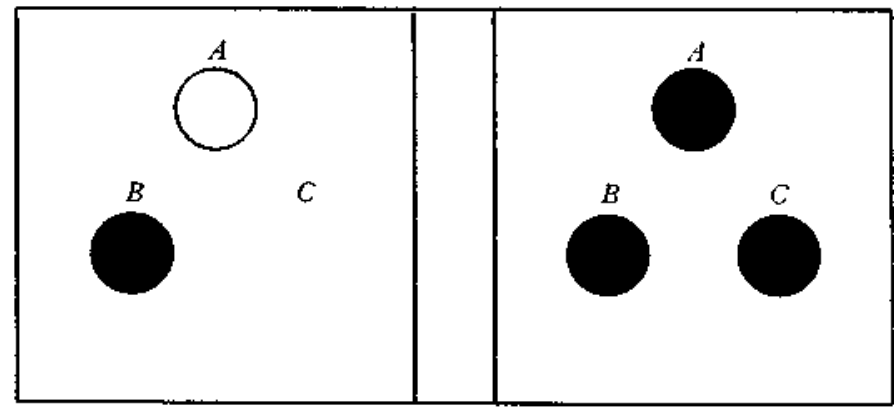

Fig. 1. Stereoscopically the disk 4 seems considerably brighter than $C$, while $B$ and $C$ do not appear very different in brightness.

Fig. 1 illustrates binocular brightness interaction. If one looks stereoscopically at the three disks $A, B$ and $C$ in Fig. 1, and compares the brightness of these disks, most people have the impression that $A$ is considerably brighter than $C$. For the centre of the disks, however, the stimulation of the eyes is identical for $A$ and for $C$; the brightness impressions are nevertheless different because of the contour in the left field of $A$. On the other hand, most observers do not see a difference in brightness between $B$ and $C$, notwithstanding the fact that the stimulus-object on the left is darker for $B$ than for $C$. It thus appears that different stimulation of the eyes may result in nearly equal impressions of brightness, when there are differences in contours.

The literature on binocular brightness interaction is of two main kinds. In studies of one kind, brightness interaction has been investigated as related to absolute 
thresholds of vision; the question then is whether the absolute threshold in one eye is dependent upon stimulation of the other eye on a corresponding area. Generally, no interaction of this sort has been reported, or only a very slight one (Graham, 1930, 1931 ; Crawford, 1940; Pirenne, 1943; Galifret, 1954). Bouman (1955), in his summary of the evidence, arrived at the same conclusion, but showed that there is a large rise of threshold for one eye within a short time (about $0.4 \mathrm{sec}$.) after the onset of stimulation of the other eye.

Studies of the second type have been concerned with brightness averaging, i.e. the apparent binocular brightness when the left and the right eye are exposed to different luminances (Panum, 1858; Fechner, 1861; Aubert, 1865; Hering, 1865; Sherrington, 1908; De Silva \& Bartley, 1930; Graham, 1931; Fry \& Bartley, 1933; Ivanoff, 1947; Fiorentini \& Radici, 1961; Treisman, 1962). Two phenomena of brightness interaction which at first sight are contradictory emerge from these studies. First, there is 'Fechner's paradox': an object of some luminance is observed binocularly but with a neutral filter in front of one of the eyes. If this eye is then closed, the brightness of the object seems to increase, although the total amount of stimulation is decreased. The second phenomenon is called brightness summation: unequal stimulation of the two eyes produces a binocular brightness which is intermediate between the monocular impressions. A better description of this phenomenon seems to be brightness averaging. The term summation has come into use, because the increase of luminance in one eye raises the binocular brightness impression.

Brightness averaging has been studied rather extensively in the past century by Panum (1858), Fechner (1861), Aubert (1865), Hering (1865) and others. Sherrington (1908) and De Silva \& Bartley (1930) have reported systematic measurements during this century. Other studies have been confined to demonstrations (Fry \& Bartley, 1933 ; Ivanoff, 1947). The most extensive measurements are those by Aubert (1865, p. 286). However, his procedure may be eriticized since he compared binocular stimuli with a monocular one, the luminance of which had to be adjusted by the observer by means of an episcotister. Given the Fechner paradox, it would be better if a binocular test stimulus were compared with a binocular comparison stimulus. This is what Sherrington did $(1908$, p. 375), but he measured the binocular brightness of five luminance pairs only. Moreover, Sherrington had the test field for one eye constant while the luminance for the other eye varied, and the observer had to adjust the comparison field with equal luminance for both eyes. As a consequence the resulting data do not give an equibrightness curve, i.e. different pairs of left and right luminances which are perceived as equal to a comparison field with constant and identical brightness for both eyes. The same applies to De Silva and Bartley's study. They kept the right field at a constant value, and used seven luminance values for the left test field. Their comparison field usually had a large spatial separation from the test field which was presented simultaneously. This introduces extra variability into the matching behaviour. In the present investigation, a number of equibrightness curves have been determined with a new matehing technique. 
LEVELT. W. J. M.. Binocular brightness averaging and contour information. British Journal of

Psychology, 56:1 (1965:Feb.) p.1

Binocular brightness averaging and contour information

EXPERIMENT I

Binocular brightness averaging

A sensible way of collecting data for equibrightness curves is to have the observer adjust a binocular test field in which the luminance for one eye is fixed by the experimenter, and that for the other eye adjusted by the observer until it produces the same brightness impression as a binocular comparison field with equal luminances for both eyes. A further requirement is that comparison and test fields are projected on corresponding retinal areas, which is the case when both stimuli are centrally fixated.

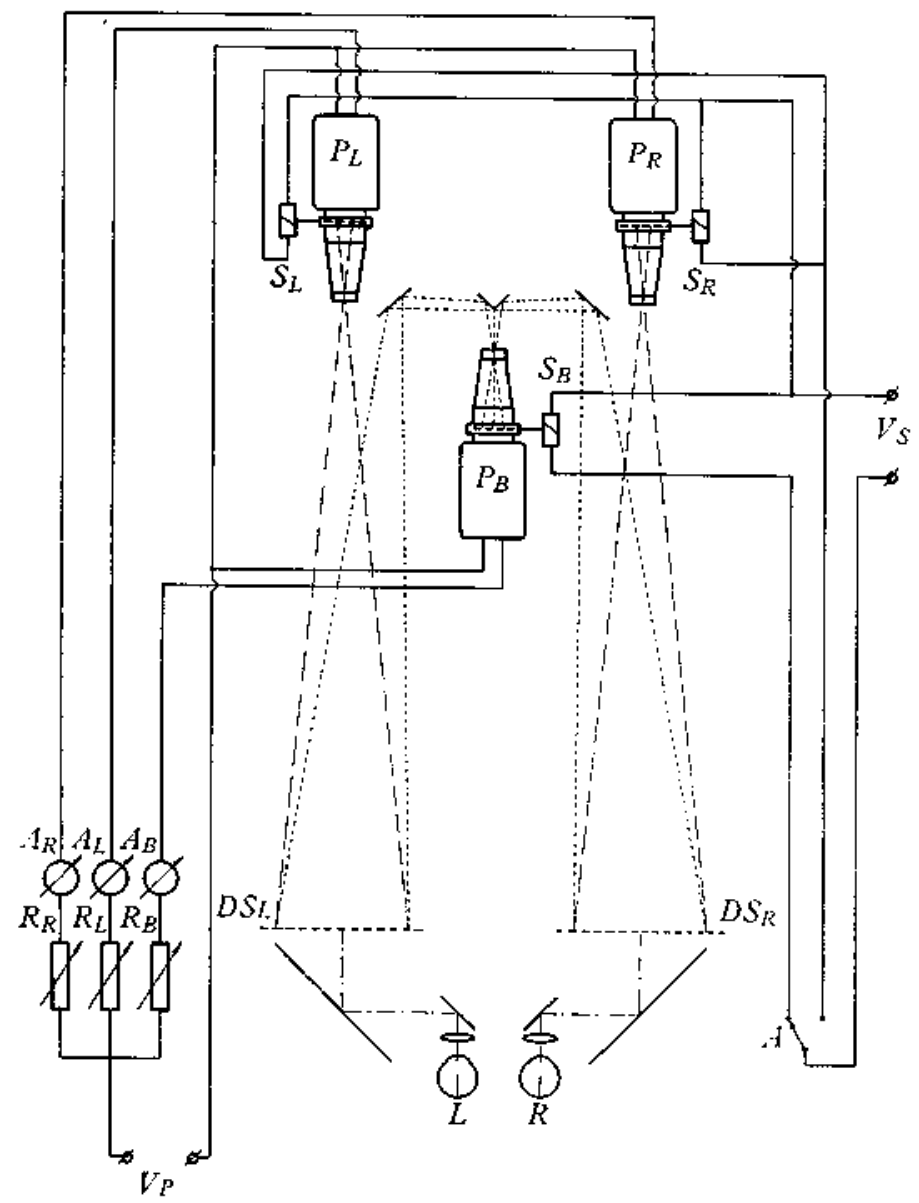

Fig. 2. Arrangement of the apparatias (see text, Expt. I).

Apparatus

Method

The apparatus is schematically represented in Fig. 2. The light sources for the test field are two $150 \mathrm{~W}$. Prado projectors $P_{L}$ and $P_{R}$, the current supply of which can be regulated by two variacs $R_{L}$ and $R_{R}$. They throw light on the diffusing sereens $D S_{L}$ and $D S_{R}$ in front of which masks can be mounted with holes of the proper size. The projector $P_{B}$ is used for the comparison field where equal luminance for both screens is needed. This is achieved by splitting the light beam from $P_{B}$ by means of a prism and two surface mirrors. Alternation of test field and com. 
LEVELT. W. J. M.. Binocular brightness averaging and contour information. British Journal of Psychology, 56:1 (1965:Feb.) p.1

parison field is regulated by a mechanism $A$, so that if the shutters $S_{R}$ and $S_{L}$ are closed, $S_{B}$ is synchronously opened, and vice versa. The images on $D S_{L}$ and $D S_{R}$ are projected to the eyes by means of two surface mirrors and two prisms. Lenses in front of the eyes serve to produce accommodation at infinity. Artificial pupils of $1 \mathrm{~mm}$. diameter are used. The observer's head is supported by a chin rest. Supply voltage was stabilized. Luminances were carefully calibrated, without artificial pupils, to a precision of within $5 \%$.

\section{Procedure}

The stimuli which were used in this experiment are shown in Fig. 3. They were circular disks subtending $3^{\circ}$ of visual angle against a black background. The luminance of the two disks in the comparison field was set at a chosen value by the experimenter. The luminance of the right test field was increased in little steps, specified below, starting at zero. At every atep the observer: had to adjust the luminance of the left field until the binoeular brightness impression was equal to that of the comparison field. The step-wise increase of luminance in the right test field was continued as long as a match remained possible. Then measurements were made similarly for increasing values of the left test field luminance and the observer had to adjust the right ont.

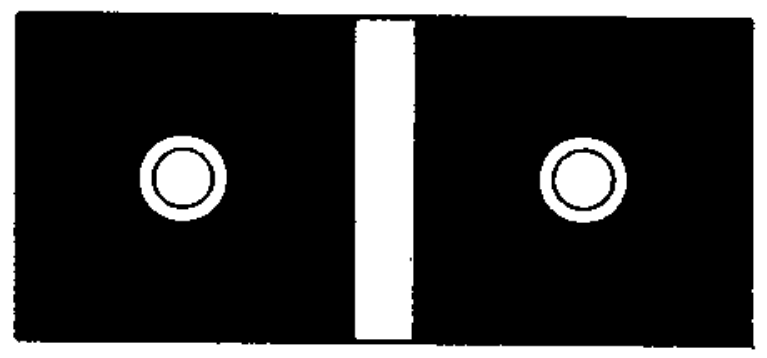

Fig. 3. Stimuli used to eletermine equibrightness curves. In Expt. I the eoncentric circles were absent when the data of Fig. 4 were collected, but were present in both monocular fields of both test and comparison fields for Fig. 5; in Expt. II, a circle was present in only one monocular field of both test and comparison fields. The visual angles substended were: circular disks, $3^{\circ}$; concentric eireles, $2^{\prime}$ in diameter and $3^{\prime}$ in thickness.

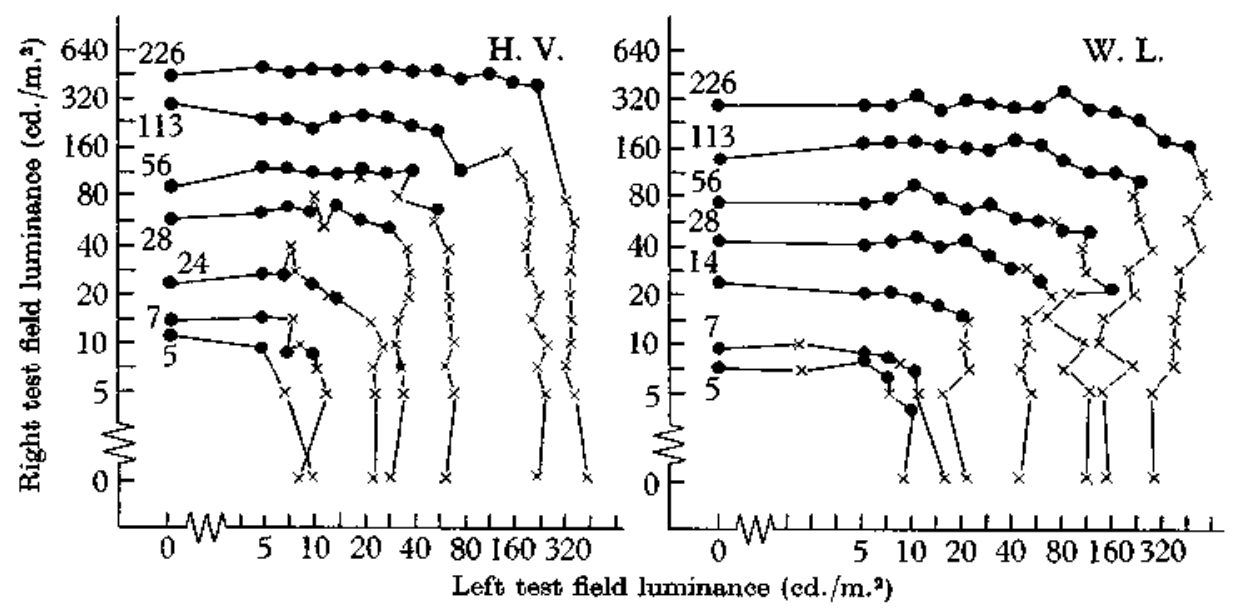

Fig. 4. Equibrightnegs curves for two observers, H, V, and W. L. The level of the comparison feld luminance is shown against each curve. The luminence of the test field was increased in logarithmic steps of $1.4 \mathrm{~cd} . / \mathrm{m}^{2}$ from $5 \mathrm{~cd} . / \mathrm{m}^{2}$ upwards; $x$ shows that the observer adjusterl the luminance of the left test field and that he adjusted that of the right test field. 


\section{Binocular brightness averaging and contour information}

Series of measurements were obtained for various levels of luminance of the comparison field. The observer could change from the test field to the comparison field and vice versa by manipuating a button, and was free to do so as often as he wanted.

\section{Results}

The restlts, in terms of equibrightness curves, are given in Figs. 4 and 5. Fig. 4 refers to measurements from a series in which the luminance of the field was increased in logarithmie steps of 1.4 from $5 \mathrm{~cd} . / \mathrm{m} .{ }^{2}$ upwards. Fig. 5 gives data for series with linear increases of the test field luminance in steps of $2 \mathrm{~cd} . / \mathrm{m} .^{2}$ from zero. First, as a check on the validity of the matching procedure, one may note that where the observer makes adjustments giving equal luminances for the test fields, their value is in close agreement with that of the comparison field; test and comparison field are identical in these cases.

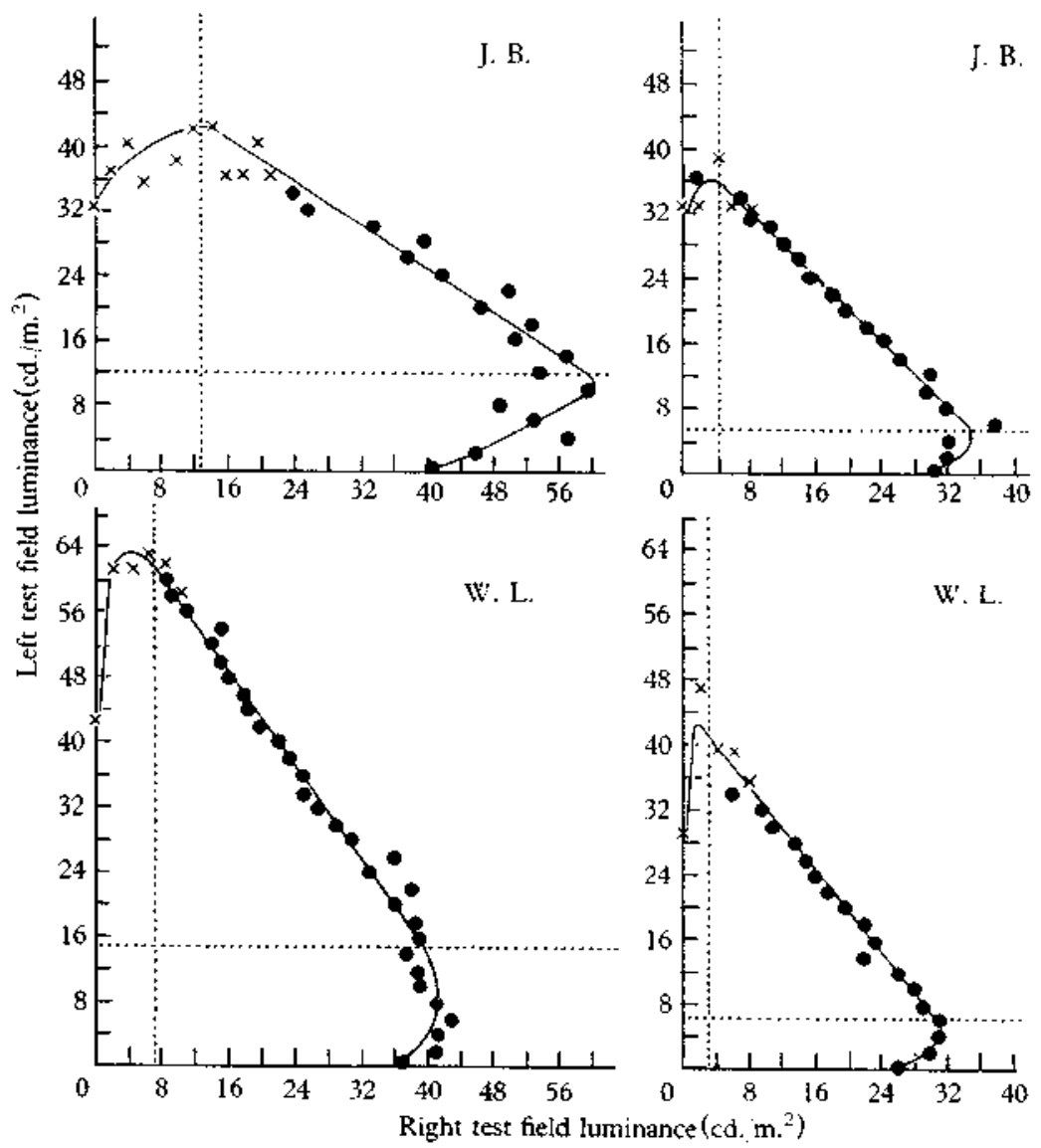

Fig. 5. Equibrightness curves for two observers, J. B. above and $W . L$. below. Two levels of comparison field luminance were used: $30 \mathrm{~cd} . / \mathrm{m} .^{2}$ on the left in each case, and $20 \mathrm{~cd} . / \mathrm{m} .^{2}$ on the right. The luminance of the test field was increased linearly in steps of $2 \mathrm{~cd} . / \mathrm{m} .{ }^{2}$ from zero; $x$ shows that the observer adjusted the luminance of the left test field and that the observer adjusted the luminance of the right test field.

The general trend of the equibrightness curves is clearest in Fig. 5. The functions are linear for test field luminances higher than a value which is indicated in each 
figure. The slope of the line is different for the two observers. It is a plausible interpretation that the slope is dependent upon eye dominance; the steep curves for W.L. are for an observer with strong dominance of the right eye; the observer J.B. does not show clear dominance. A difference in sensitivity between the two eyes might be an alternative hypothesis. However, when both subjects were tested on the Haag-Streit Adaptometer during 3 min. after foveal adaptation to $2,000 \mathrm{~cd} . / \mathrm{m} .{ }^{2}$, neither subject showed a difference in foveal sensitivity between the two eyes.

The linear portions of an equibrightness curve can be expressed as : $w_{l} E_{l}+w_{r} E_{r}=O$. Here $E_{l}$ and $E_{r}$ are luminances of left and right test fields, respectively; and $w_{l}$ and $w_{r}$ can be interpreted as weighting coefficients which account for eye dominance. For the point $E_{l}=E_{r}=E$ we have: $\left(w_{l}+w_{r}\right) E=C$; therefore it is only natural to choose $w_{7}+w_{r}=1$, so that $C=E_{b}$, the luminance of the comparison field. The fact that the curves are linear, disregarding the tails, implies that binocular brightness averaging can be simply described as an averaging of energies, and thus far there is no special reason to claim that brightness averaging is a matter of averaging of 'sensations'. The latter claim was made by Aubert and later on by Sherrington. Sherrington went as far as to speculate that the sensorium of the right eye is completely separated from that of the left eye. Whatever the truth of this, the present curves suggest that the binocular brightness impression does not result from simple averaging of monocular sensations: it is known from psychophysical studies that monocular and normal binocular subjective brightness are non-linear functions of stimulus energy. Irrespective of whether this is a logarithmic function (Fechner), or a power function (Stevens), or any other non-linear function, the result could never be that binocular brightness is a linear function of its monocular components if sensations were merely averaged. The experimental error in the present measurements evidently tolerated slight deviations from linearity, but even a function of power $1 / 2$ produces a bend, similar in all curves. Therefore, if binocular brightness were a matter of combining sensations, the results suggest that they would have to be combined in a more complicated manner: the resulting binocular brightness would have to be the same as if energies were directly averaged.

It is concluded, therefore, that binocular brightness is constant if a weighted sum of monocular energies is constant; the weighting coefficients add to unity and are constant for an individual observer. This rule is not valid if one of the monocular luminosities is low.

\section{EXPERIMENT II}

\section{Monocular contour information}

The following experiments were the main experiments of this study and they were undertaken to assess the role of monocular contour information in binoeular brightness averaging. The first experiment is an obvious extension of the experiment described earlier. Equibrightness curves were again determined, with the difference that a concentric circle, subtending $2^{\circ}$, was present in one of the monocular fields of both the test field and the comparison field (cf. Fig. 3). The observer was instructed to match the fields for the interior of the circles. The comparison field was kept at $30 \mathrm{~cd} . / \mathrm{m} .^{2}$. In all other respects the procedure was the same as described before. 
LEVELT. W. J. M., Binocular brightness averaging and contour information. British Journal of

Psychology, 56:1 (1965:Feb.) p.1

Binocular brightness averaging and contour information

Results

Fig. 6 shows results for circles in the left fields, and Fig. 7 for circles in the right fields. The curves are again linear, except for the tails, and pass through the point $E_{l}=E_{r}=30$. This means that they may again be described by the function*

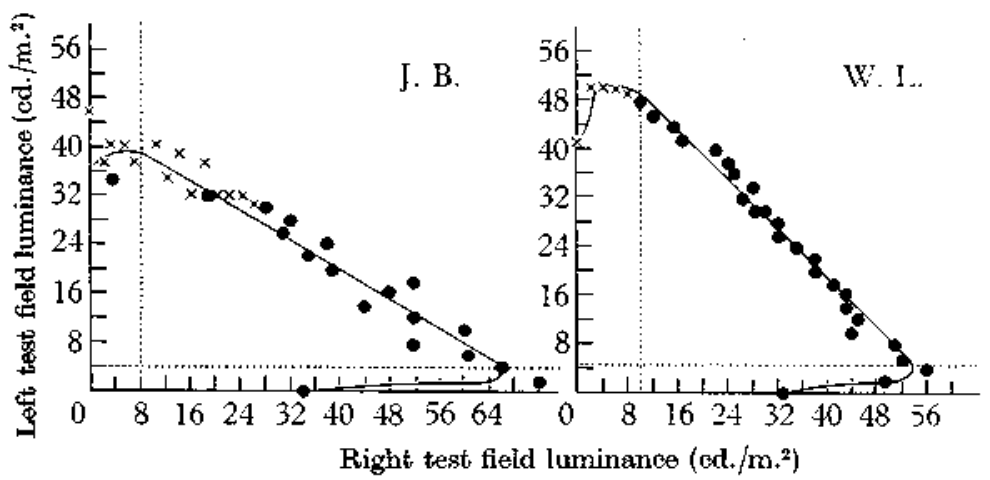

Fig. 6. Equibrightness eurves for two observers, J. B. and W. L., with a circle present in the left monocular field of both the test field and the comparison field (see Fig. 3). The luminance of the comparison field was $30 \mathrm{~cd} / / \mathrm{m}^{2}{ }^{2}$.

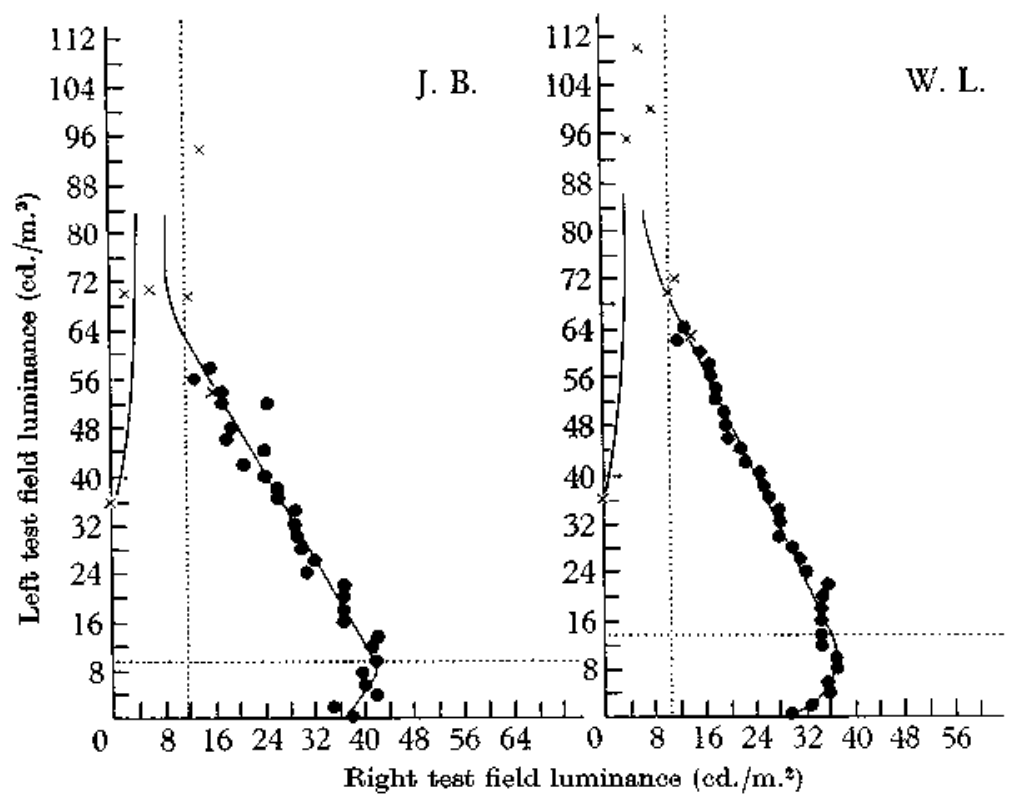

Fig. 7. Equibrightness curves for two observers, J. B. and W. L., with a circle present in the right monocular field of both the test field and the comparison fiekl (see Fig. 3). The Juminance of the comparison field was $30 \mathrm{cl} . / \mathrm{m} .{ }^{2}$.

* The circle in one of the monocular comparison fields serves to malke the matehing process easter for the observer. The question arises, however, of whother $E_{b}$ retains the same meaning when $C$ is equated to it. In other words, for equal stimulation of both eyes, docs the binocular brightness impression change if a circle is eliminated from one of the monocular fields? This seems very improbable; the assumption was ehecked by having an observer compare a pair of fields of luninance $30 \mathrm{~cd} . / \mathrm{m} .{ }^{2}$. both containing a $2^{\circ}$ circle, to an adjustable pair with a circle in the left field only. Ten adjustments were made; their mean value was $30 \cdot 6,0 \cdot 49$. The assumption may be maintained, therefore. 
$w_{l} E_{l}+w_{r} E_{r}=C=E_{b}$, where $w_{l}+w_{r}=1$. The only difference between the three curves from each observer is in their inclination (see Figs. 5-7). This can be expressed as follows: the weighting coefficient for the eye in which contour information is present is increased relative to the coefficient for the other eye. This may be called the law of complementary shares, after Hering.* It simply means that if $w_{l}$ increases $w_{r}$ decreases, and vice versa, so that their sum remains unity.

The law enables a comment to be made on the tails of the equibrightness curves, where one of the monocular components has a Iow value. It is elear that if the luminance of one test field, the left one say, is below threshold, contour information is present in the right field only. Therefore, for these low values of $E_{l}, w_{r}$ will increase at the cost of $w_{1}$, and so a change in slope of the curves at the tails is predicted (Figs. 4-7). It is not possible to give the precise function of the non-linear parts of the curves; in particular, the luminance values at which the weighting coefficients start changing are difficult to estimate. They are probably not at the threshold value for one eye, but at a value at which the contour information has 'faded out'sufficiently.

\section{EXPERIMENT III}

\section{Change in weighting as dependent upon distance from contour}

A monocular contour increases the weighting coefficient for the luminance in the corresponding eye. The next question is whether the effect of a contour is local or general, i.e. is there an increase in $w$ for the whole monocular visual field, or is the increase limited to a region in the immediate environment of the contour?

\section{Method}

The stimuli for this experiment are shown in Fig. 8. They were four pairs of patterns, subtending $5^{\circ}$, containing circles. These stimuli were used for both the test and the comparison field. The difference was again that in the test field luminances were unequal, $250 \mathrm{~cd} . / \mathrm{m} .{ }^{2}$ for the left eye and $25 \mathrm{~cd} . / \mathrm{m} .{ }^{2}$ for the right eye, whereas in the comparison field they were equal. In this experiment the observer had to adjust the comparison field until the brightness in the centre of the upper cirele looked equal for both fields. Four observers served in this experiment. The four different stimulus conditions were given to them in an order which was varied according to a Latin square design.

\section{Results}

Table 1 gives the results in terms of the adjusted luminance in the comparison field. An analysis of variance reveals significant differences between the conditions. First, the $C$ pair gave a higher value than the $D$ pair $(P<0.001)$. For the $C$ pair the contour was in the more luminous (left) test field, whereas for the $D$ pair it was in the less luminous (right) field; this result therefore confirms the argument that has been advanced that contour information influences the weighting coefficient. Secondly, in both $A$ and $B$ pairs one circle is present in the more luminous left field, and one circle in the dimmer right field. If the influence of the contour extends to the whole field, the matchings for the upper circle area in $A$ and $B$ would not be expected to be different. If, however, only the direct environment of the contour is effective, the comparison field for $A$ should be adjusted to a higher luminance than that for $B$.

* Hering (1865, pp. 308 ff.) suggested this lew (Gezetz des complementären Antheils der Netzhäute am Schraume) for any kind of binocular interaction, without giving a quantitative apecification. 
LEVELT. W. J. M., Binocular brightmess averaging and contour information . British Journal of Psychology, 56:1 (1965:Feb.) p.1

Binocular brightness averaying and contour information

Table 1 shows that the latter is the case $(P<0 \cdot 005)$. The differences between $A$ and $C$, and $B$ and $D$ do not reach significance. The conclusion is, therefore, that the weighting coefficient is increased only for the immediate neighbourhood of contours, not for the visual field as a whole.

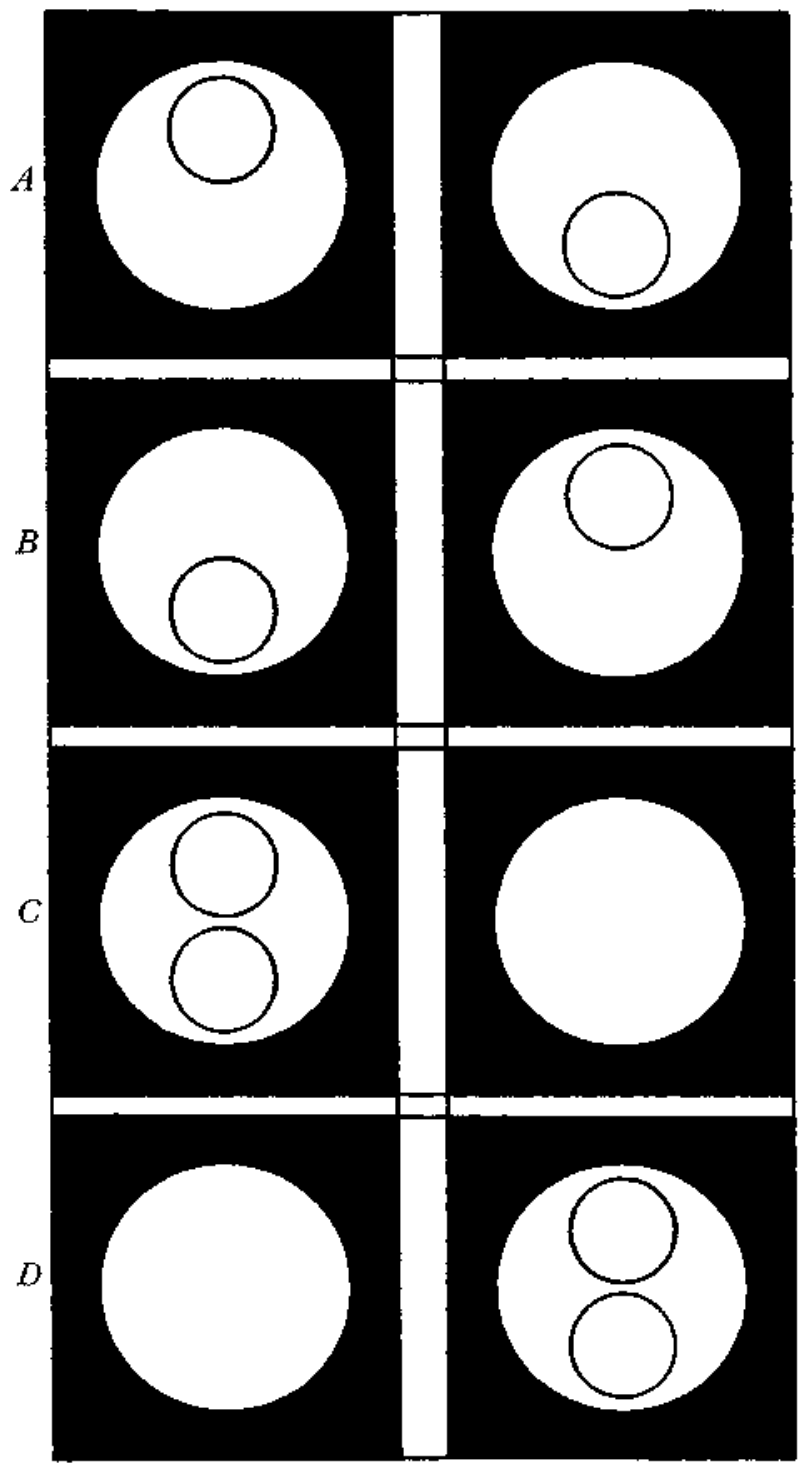

Fig. s. F'our pairs of stimuli which were used to test whether the influence of contrours was loral ur general. The sance stimuli were used for both the test and the comparison fields. The dianter of the risks was $5^{c}$ of visual angle. Test field luminances were always $250 \mathrm{~cd} . / \mathrm{m} .{ }^{2}$ for the left field and $25 \mathrm{ed} / \mathrm{m}^{2}$ for the right field. The obserser artjusterd the luninanse of the ares within the upprer circle only of the comparison firld. 
LEVELT. W. J. M.. Binocular brightness averaging and contour information. British Journal of Psychology, 56:1 (1965:Feb.) p.1

Table 1. Comparison field adjustments in cd. $/ \mathrm{m}^{2}$ of four observers for the conditions of Fig. 8, Expt. III

\begin{tabular}{|c|c|c|c|c|}
\hline \multirow[b]{2}{*}{ Observer } & \multicolumn{4}{|c|}{ Condition } \\
\hline & $A$ & $B$ & $C$ & $D$ \\
\hline I & 202 & 53 & 195 & 70 \\
\hline 2 & 180 & 43 & 190 & 50 \\
\hline 3 & 180 & 57 & 223 & 80 \\
\hline 4 & 202 & 117 & 270 & 104 \\
\hline Means & 191 & $67 \cdot 5$ & 219.5 & 76 \\
\hline & & IME & & \\
\hline
\end{tabular}

The next question follows immediately: to what value does $w$ inerease at the fixation point if the distance between the fixation point and the contour is made smaller and smaller? In other words, this question refers to the spatial amplitude of variation in $w$.

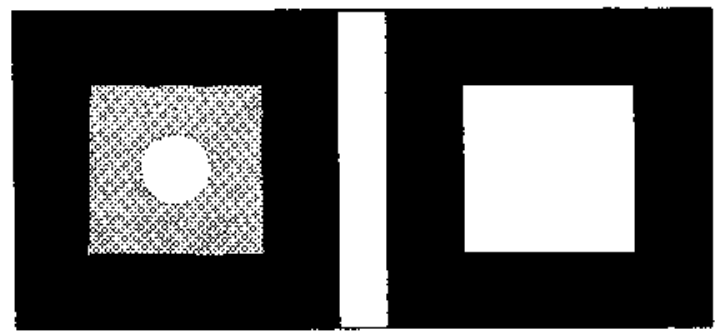

Fig. 9. Stimuli used to determine $w_{t}$ as a function of field size. The squares were $14^{\circ} \times 14^{\circ}$ of visual angle; four sizes of central disk in the left feld were used $: 1^{\circ}, 3^{\circ}, 5^{\circ}$ and $7^{\circ}$. The patterns of the left and right test and comparison fields were the same; see text for details of the luminance relations.

\section{Method}

The stimulus conditions for this experiment are shown in Fig. 9. The right test field was a square of $14^{\circ} \times 14^{\circ}$. Its luminance was fixed at $100 \mathrm{~cd} . / \mathrm{m} .^{2}$. The right comparison field was identical, but its brightness was adjugtable by the observer. The left test field consisted of two parts: a central disk of variable size, with luminance fixed at $12 \mathrm{~cd} . / \mathrm{m} .^{2}$, and a surrounding field of $14^{\circ} \times 14^{\circ}$ at luminance $3.7 \mathrm{ed} . / \mathrm{m}{ }^{2}$. The left comparison field had the same pattern; the luminance of the central disk was always the same as that adjusted by the observer for the right field, and the luminance of the surrounding area was always $1 / 3.25$ of that of the central disk. The observer had to adjust the comparison field until the brightness of the central diak appeared equal for both test field and comparison field, while fixating the centre of the disk.

Four sizes of disk were used $: 7^{\circ}, 5^{\circ}, 3^{\circ}$ and $1^{\circ}$ of visual angle. The conditions were presented according to a Latin square design. Two groups of four observers took part in the experiment.

In this experiment boundaries between two different luminances were used instead of contours on a uniform background. The reason was that it is quite impossible to make a trustworthy brightnese match of the area within a contour of $1^{\circ}$ on a relatively large, uniformly illuminated background, when instructed to neglect the brightness of this surrounding field. The problems of brightness contrast in this stimulus arrangement were minimized in two ways. The central disk was brighter than the surrounding area, and the brightness contrast effect is only great for a stimulus shown against a more luminous background. Moreover, the ratio between disk and surrounding luminance was equal for test and comparison fields and constant throughout the experiment. 


\section{Results}

Individual values of $w$ have been calculated for the four disk sizes. The results are given in Table 2. Analysis of variance shows that $w_{l}$ increases with decreasing diameter of the disks; the regression is significant $(P<0.001)$. In Table 2 , it is seen that at $1^{\circ}$ for observers 3,6 and $8, w$ approaches the unit value as closely as adjustment errors permit. In view, moreover, of the inereasing tendency apparent in the mean values of $w$ for decreasing size of the disk, the data strongly suggest that in the immediate nejghbourhood of a monocularly presented contour, binocular brightness is exclusively determined by the luminance of this monocular field. The strongly localized contour effects in the former experiment (Table 1), compared with the relatively high values of $w$ for all angles in Table 2, suggests that variation of $w$ is limited by the presence of a contour. Furthermore, a boundary may be more effective than a contour.

Table 2. Values of $w_{l}$ for eight observers and four sizes of disk

\begin{tabular}{crrrr} 
& \multicolumn{3}{c}{ Size of disk (visual angle) } \\
\cline { 2 - 5 } Observer & $1^{\circ}$ & $3^{\circ}$ & $5^{\circ}$ & $7^{\circ}$ \\
1 & .835 & .786 & .741 & .798 \\
2 & .956 & .873 & .941 & .875 \\
3 & .997 & .981 & .974 & .968 \\
4 & .911 & .830 & .765 & .792 \\
5 & .939 & .914 & .824 & .801 \\
6 & .990 & .900 & .881 & .864 \\
7 & .824 & .810 & .790 & .773 \\
8 & 1.002 & .998 & .941 & .937 \\
Means & .932 & .887 & .857 & .851
\end{tabular}

Fechner's paradox can now be understood as follows. If a neutral filter is placed before the right eye, $E_{b}=w_{l} E+w_{r} t E=E\left(w_{l}+w_{r} t\right)$, where $t$ is the transmission of the filter. If the right eye is closed, $w_{l}$ equals unity, so that $E_{b}^{\prime}=E$. Since $w_{1}+w_{r}=1$, and $t<1$, it follows that $w_{l}+w_{r} t<1$, and therefore $E_{b}^{\prime}>E_{b}$. Hence the apparent brightness increases if the right eye is closed. This argument implies, however, that the assumption that $w_{l}+w_{r}=1$ remains valid for monocular observation. There is some evidence that this is not true without qualification. In fact, one may interpret the data for $E_{r}=0$ (Figs. 6 and 7 ) as an indication that $w_{l}+w_{r}<1$ there also, since $E_{b}^{\prime}<E_{l}$ (similarly for $E_{l}=0$; comparable instances are given by De Silva \& Bartley, 1930 as argument for brightness summation). However, two considerations apply.

(a) Matching a monocular and a binocular brightness appears to be a rather unstable affair. Day-to-day variability is high. Observers have a feeling that at every new experimental session some arbitrary criterion is chosen, a feeling that is absent for binocular-binoeular matehings.

(b) To check whether the assumption that $w_{l}+w_{r}=1$ remains valid for monocular observation, a very small test field (or comparison field) has to be used in order to ensure that the field as a whole is sufficiently close to contour information for its weighting coefficient to approach unity. Closeness to contour information may be relevant; in Fechner's original experiments the paradox was absent for filter transmission values closely approaching unity (Fechner, 1861, p. 420), indicating that $E_{b}^{\prime}<E$ in the above sense. This follows necessarily if $w_{l}<\mathbf{1}$, because $E=w_{l} E_{b}^{\prime}<E$. 
According to Table $2, w$ is less than unity at a great distance from the contour, and this was precisely Fechner's situation because he instructed his subjects to look at the blue sky through the filter.

For these reasons it does not seem necessary to reject the law of complementary shares for the case of monocular stimulation. In addition, the explanation of Fechner's paradox would not be invalidated if the law of complementary shares had to be qualified. A good choice of the transmission of the filter might always compensate for minor deviations from the law.

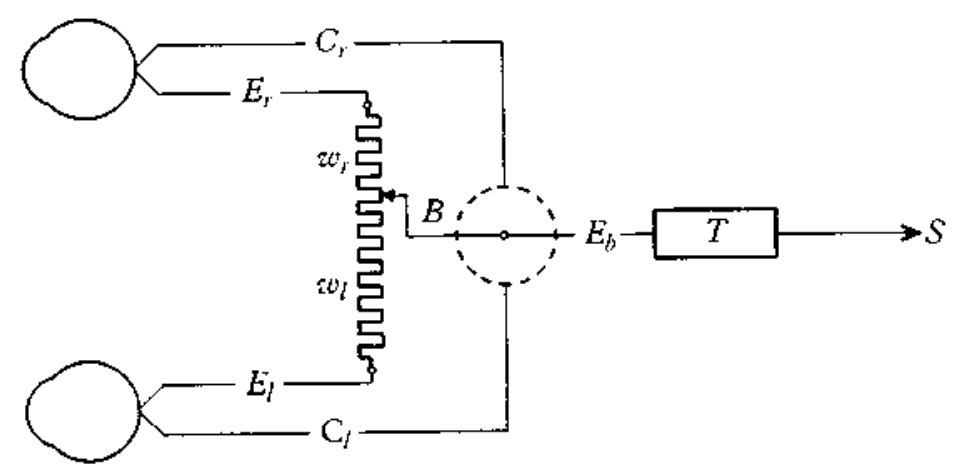

Fig. 10. Schernatic representation of the interaction of monocular contours and luminances in binocular brightness averaging; text for sletails.

\section{CONCLUSIONS}

The general conclusions from this study may be summarized with the help of Fig. 10. This diagram is intended as a mnemonic device, without further pretensions, and is drawn as an electrical flow-diagram. Luminosities $E_{l}$ and $E_{r}$ stimulate the eyes and may be thought of as voltages in the electrical metaphor. They are weighted by the balance $B$, a potentiometer in electrical terms, and their weighted sum $E_{b}$ is the basis for the binocular impression of brightness; $w_{l}$ and $w_{r}$ become variable resistances with a constant sum, representing the law of complementary shares; as a result, the current in the channel past $B$ is $E_{b}=w_{l} E_{l}+w_{r} E_{r}$. The weighting coefficients, $w$, depend upon contour information, as indicated by the channels $c_{l}$ and $c_{r}$ to $B$, which determine the position of the potentiometer. If contour information is similar for both eyes, the weighting coefficients are determined by eye dominance only; in the absence of eye dominance they are both equal to one-half. If contour information is present for one eye only, the weighting coefficient for this eye increases, up to a maximum of unity, with a corresponding decrease in the coefficient for the other eye according to the law of complementary shares. The whole mechanism applies to a small area of the visual field only; for a different area a similar mechanism must be postulated for which the parameters may be different.

If the binocular brightness impression is a function of averaged energies, the "translation', $T$, of the weighted energies into a binocular brightness sensation has been simply located in the diagram beyond the averaging process, and $T$ can be imagined as a device with some non-linear amplitude transfer characteristic. At every point 
of the visual field, the apparent brightness is thus determined by the left and right eye luminances at that point, and by left and right eye contours near the point.

The implications of the approach for the mechanism of binocular rivalry are considered in conclusion. Rivalry occurs, generally, when non-corresponding contour information is given. Rivalry is not a matter of the monocular visual fields as a whole; parts of one field may enter into rivalry with parts of the other field. In terms of Fig. 10, if non-corresponding contours are present near an area $T$, a conflict results in the partition of the weights. The contour in the left eye produces a tendency for $w_{l}$ to increase; the non-corresponding contour in the right eye, in its turn, will produce a tendency for $w_{r}$ to rise. An increase of both $w_{l}$ and $w_{r}$ would evidently violate the law of complementary shares. Apparently this conflict is resolved in such a way that one tendency triumphs over the other for some time, after which the other tendency becomes victorious. The law of complementary shares is thus saved by an alternating process. Evidence for this process will be given in a subsequent report.

The author is grateful to Prof. J. P. van de Geer for stimulating this research and correcting the text, and to Prof. M. A. Bouman for eritically reading and discussing the manuscript.

\section{RefERences}

Aubert, H. (1865). Physiologie der Netzhaut. Breslau : Morgenstern.

Bounan, M. A. (1955). On foveal and peripheral interaction in binocular vision. Opt. Act. 1, $177-83$.

CRAWFORD, B. (1940). Oeular interaction in its relation to measurements of brightness threshold. Proc. Roy. Soc. B, 128, 552-61.

De Silva \& Bartley, S. H. (1930). Summation and subtraction of brightness in binoculaw perception. Bril. J. Psychol. 20, 241-50.

Fechmer, G. J'H. (1861), U̇ber einige Verhältnisse dos binoeularen Sehens. Abh. sächs. Ges. (Akad.) Wiss. 7, 337-564.

Fiorentini, A. \& Radici, 'l'. (I961). Sur un effet d'interaction binoculaire. Vision Res. 1, $244-52$.

Fry, G. A. \& Barteey, S. H. (1933). The brilliance of an object seen binoctlarly. Amer. $J$. Ophthalmol. 16, 687-93.

Gacrfret, Y. (1954). Contributions à l'étude du problème de l'interaction des stimulations périphériques binoculaires. C.R. Soc. Biol., Paris, 148, 215.

Graham, C. H. (1930). An investigation of binocular summation. I. The fovea. J. Gen. I'sychol. 3, 494-510.

GrabaM, C. H. (1931), An investigation of binocular summation. II. The periphery. .7. Gen. Psychol. 5, 311-27.

HERING, E. (1865). Beiträge zur Physiologie. Leipzig: Engelmann.

Ivanoff, A. (1947). Au sujet paradoxale de Fechner. Acal. Sci. Opt. Physiol. Sénce du 19 mai, 1947.

Pandm, P.L. (1858). Physiologische Untersuchangen über das Selen mitzweiAugen. Kiel : Sehwerst PrRenne, M. H. (1943). Binocular and uniocular threshold of vision. Nature, Lond., 152, 698. SHerrington, C.S. (1908). The Integrative Action of the Nervous System. London: Constable.

Treisman, A. (1962). Binocular rivalry and stereoscopie depth perception. Quart. J, Exp. Psychol. 14, 23-37.

(Manuscript received 28 January 1964) 